\title{
Meta-Analysis of the Genetic Association between PTPN22 and CTLA-4 Variants and Risk of Uveitis
}

\author{
Jun Zhang ${ }^{\mathrm{a}}$ Peng Zhou ${ }^{\mathrm{b}}$ Shuqiong $\mathrm{Hu}^{\mathrm{c}}$ Jian $\mathrm{Qj}^{\mathrm{d}}$ \\ ${ }^{a}$ Chongqing Key Laboratory of Ophthalmology, and Chongqing Eye Institute, The First Affiliated Hospital of \\ Chongqing Medical University, Chongqing, PR China; 'bState Key Laboratory of Biocontrol, School of Life Sciences, \\ Sun Yat-sen University, Guangzhou, PR China; 'Wuhan Aier Eye Hospital of Wuhan University, Wuhan, PR China; \\ ${ }^{\mathrm{d} D e p a r t m e n t}$ of Ophthalmology, Daping Hospital, Army Medical University, Chongqing, PR China
}

\section{Keywords}

Uveitis · PTPN22 $\cdot$ CTLA-4 $\cdot$ Polymorphism $\cdot$ Meta-analysis

\begin{abstract}
Background: Though the risk of protein tyrosine phosphatase nonreceptor 22 (PTPN22) and cytotoxic T-lymphocyte antigen-4 (CTLA-4) genetic variants with uveitis have been developed, the combined results still remain uncertain and controversial. Objectives: The aim of this study was to perform a meta-analysis to estimate the precise association of PTPN22 (rs2488457 and rs2476601) and CTLA-4 (rs231775, rs5742909, rs4553808, and rs3087243) polymorphisms with uveitis susceptibility. Method: Five electronic databases (PubMed, Embase, Web of Science, China Biomedical Database, and China National Knowledge Infrastructure) were systematically searched for relevant literature up to July 20, 2021. All statistical analyses were evaluated by Stata 12.0 software and R programming language. Results: Our metaresults indicated that PTPN22 rs2488457 conferred positive susceptibility to uveitis (odds ratio $[O R]=1.18,95 \%$ confidence interval $[\mathrm{Cl}]=1.02-1.38, p=0.029)$. In stratified analysis by ethnicity, the rs $2488457 \mathrm{C}$ allele had a growing tendency toward uveitis in the Asian region $(O R=1.21,95 \%$
\end{abstract}

Karger@karger.com www.karger.com/ore

Karger"
(C) 2022 The Author(s)

Published by S. Karger AG, Basel

This is an Open Access article licensed under the Creative Commons Attribution-NonCommercial-4.0 International License (CC BY-NC) (http://www.karger.com/Services/OpenAccessLicense), applicable to the online version of the article only. Usage and distribution for commercial purposes requires written permission.
$\mathrm{Cl}=1.00-1.45, p=0.046)$. For CTLA-4 rs231775, subgroup analysis based on ethnicity manifested a negative association among uveitis individuals in the Africa region $(\mathrm{OR}=0.25$, $95 \% \mathrm{Cl}=0.19-0.33, p<0.001)$. For CTLA-4 rs4553808, subgroup analysis by the disease type revealed that the GG genotype was associated with an elevated risk of Behcet's disease (BD) $(\mathrm{OR}=3.22,95 \% \mathrm{Cl}=1.05-9.90, p=0.042)$. Conclusions: Our research revealed that PTPN22 rs2488457 conferred strong susceptibility to uveitis in general, especially in the Asian region. CTLA-4 rs231775 conveyed protection against uveitis in African populations, and CTLA-4 rs4553808 displayed an increased risk of BD.

(c) 2022 The Author(s).

Published by S. Karger AG, Basel

\section{Introduction}

Uveitis, or inflammation of the uvea, is becoming a leading cause of visual loss. Uveitis can be clinically recognized with distinct phenotypes. Behcet's disease (BD) and Vogt-Koyanagi-Harada (VKH) syndrome have represented the most two common type of multisystem immune-mediated or inflammation-related uveitis [1, 2]. Although the pathophysiology of these phenotypes re-
Correspondence to:

Jian Qi, yankeqijian@ qq.com 
mains to be elucidated, they are always characterized by immune hyperreactivity, mainly affecting elements of the immune system [3]. It is generally acknowledged that environmental and genetic factors are important components of the etiologies of $\mathrm{BD}, \mathrm{VKH}$ syndrome, and other types of uveitis $[4,5]$. Moreover, there is evidence of a strong association of the HLA allele with $\mathrm{BD}$ or $\mathrm{VKH}$ syndrome $[6,7]$. However, mutations in HLA regions do not account for all uveitis individuals, indicating that nonHLA susceptibility genes also confer a genetic predisposition to these disorders.

A number of studies have revealed that an autoimmune response, in particular cellular immunity against $\mathrm{T}$ lymphocytes and tyrosinase family proteins, acts a pivotal part in the initiation and maintenance of uveitis [810]. Lymphoid tyrosine phosphatase (LYP) is a member of the protein tyrosine kinases family and expressed in $\mathrm{T}$ cells [11]. The LYP protein is encoded by the human protein tyrosine phosphatase nonreceptor 22 (PTPN22) gene on chromosome 1p13 [11], which plays a negative regulatory role in the T-cell signaling pathway. Dysfunction of PTPN22 (i.e., increased activity) suppressed the T-cell receptor (TCR) signal transduction of regulatory T cells, thus weakening the regulation function of $\mathrm{T}$ cells and leading to autoimmune diseases [12]. Many functional mutations have been described and conferred either altered susceptibility to autoimmune diseases or appeared to influence the severity and clinical outcomes [13]. The most widely surveyed single-nucleotide polymorphisms (SNPs) of the PTPN22 gene associated with uveitis were 1123G/C (rs2488457) and R620W (rs2476601).

Research has demonstrated that T-cell activation requires costimulatory signals involving $\mathrm{CD} 28$ and CD40 with B7-1 (CD80) and B7-2 (CD86) on antigen-presenting cells [14]. Cytotoxic T-lymphocyte antigen-4 (CTLA-4), a homolog of CD28, binds to CD80 and CD86 to negatively regulate T-cell activation [15]. Until now, the most shared SNPs of the CTLA-4 gene reported in association with uveitis included 49A/G (rs231775), 318C/T (rs5742909), 1661A/G (rs4553808), and CT60G/A (rs3087243).

PTPN22 and CTLA-4 genes, as susceptibility candidate locus involvement in inflammatory or immune-mediated disorders, have been commonly explored during the past decades. Quite a few meta-analyses of CTLA-4 gene polymorphisms with $\mathrm{BD}$ risk have been performed $[16,17]$; however, these research studies only focused on two SNPs (CTLA-4 rs231775 and rs5742909). Several studies have shed light on the correlation of three SNPs (CTLA-4 rs4553808, rs3087243, and PTPN22 rs2476601) with uveitis $[18,19]$. Although the relationships between
PTPN22 and CTLA-4 genetic variants with uveitis have been comprehensively reported, the combined results are controversial and ambiguous. Another weakness in previous published articles is attributed to their relatively small sample size and few ethnic origins, suggesting insufficient power and inadequate evidences. To better clarify the genetic basis for the relationship between PTPN22 (rs2488457 and rs2476601) and CTLA-4 (rs231775, rs5742909, rs4553808, and rs3087243) toward the risk of uveitis in different races, a meta-analysis has been performed.

\section{Materials and Methods}

This current meta-analysis was conducted and reported in accordance with the PRISMA guidelines [20] (see online suppl. Table 1; see www.karger.com/doi/10.1159/000521301 for all online suppl. material).

\section{Search Strategy}

A literature search of five common electronic databases (PubMed, Embase, Web of Science, China Biomedical Database, and China National Knowledge Infrastructure) was conducted to identify relevant studies published from the inception to July 20, 2021. Reference lists in the retrieved studies were also manually screened. Searches were retrieved using the following combined terms "uveitis," "PTPN22," "CTLA-4," and "polymorphism" (see online suppl. Table 2). Two researchers (J.Z. and P.Z.) reviewed reports and dismissed irrelevant studies. Once uncertain conclusion arose, it would be solved by discussion with the third author.

\section{Criteria for Identification}

The inclusion criteria for an article were as follows: (1) it focused on the association between PTPN22 and CTLA-4 genetic polymorphisms and uveitis; (2) sufficient data of the alleles and genotypes in the comparison of case-control group; and (3) it focused on human subjects. The exclusion criteria were (1) not a case-control study; (2) genotype or allele frequency could not be extracted; (3) duplicates of previous publications; and (4) metaanalyses, letters, animal studies, reviews, and editorial articles. Only the study with the largest sample size or the most recent publication was enrolled, when the authors published several studies including the same subjects.

\section{Quality Assessment}

The quality evaluation of extracted studies was also reviewed by 2 authors (J.Z. and P.Z.) using the Newcastle-Ottawa scale [21]. In the case-control study, three items to assess potential bias in genedisease associations were included: selection of cases and controls, comparability of cases and controls, and ascertainment of exposure. In the quality evaluation, each item was awarded a different number of stars: selection of cases and controls $=4$ stars, comparability of cases and controls $=2$ stars, and ascertainment of exposure $=3$ stars. A study with more than 6 stars was regarded as high quality, and the one with 9 stars was regarded as the highest quality. Disagreements were resolved by discussion with a third reviewer (S.H.). 
Fig. 1. Flow diagram of literature search and screening in current meta-analysis.

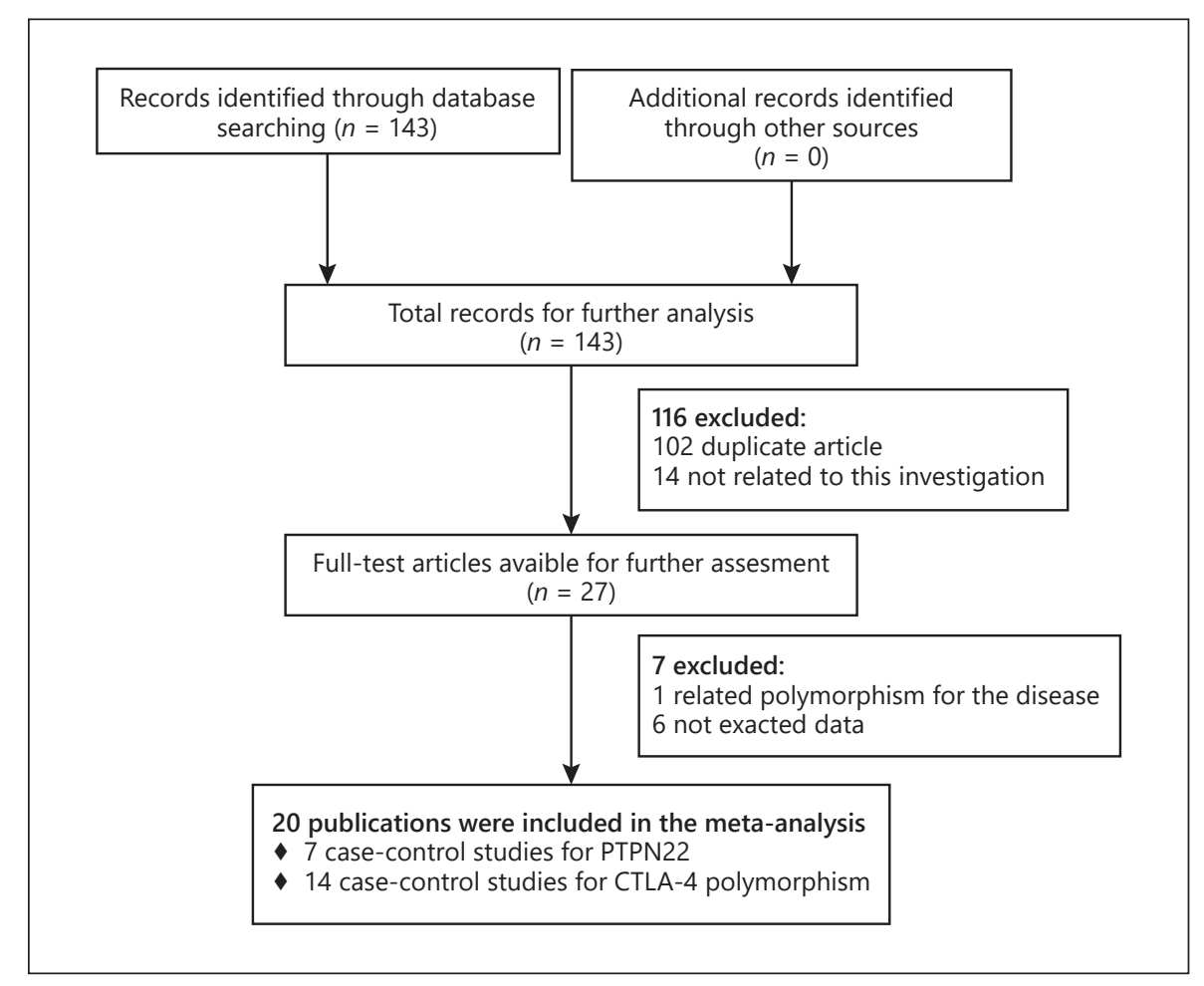

\section{Data Extraction}

Two reviewers (J.Z. and P.Z.) independently retrieved articles and extracted data from the eligible studies. The following information was collected from each record: the name of the first author, year of publication, country and ethnicity of the sample, characteristics and number of cases and controls, genotyping method, and the frequency of alleles and genotypes in cases and controls. To explore the differences between PTPN22 and CTLA-4 genetic susceptibility and various uveitis subtypes, we divided uveitis into a BD subtype and other types of uveitis. In the event of disagreement, 2 reviewers should examine the collected date and reached a consensus following a rigorous screening process. If consensus was not met, a third reviewer (S.H.) was asked to resolve the dispute in depth.

\section{Statistical Analysis}

Cochran's Q statistic was used to assess heterogeneity with a $p$ value less than 0.1 , indicating statistical significance. Quantitative $I^{2}$ was calculated to estimate inconsistency in the meta-analysis and represented the percentage of observed variability due to heterogeneity rather than chance (no heterogeneity, $I^{2}=0-25 \%$; moderate heterogeneity, $I^{2}=25-50 \%$; large heterogeneity, $I^{2}>$ $50 \%$ ) [22]. In case of no heterogeneity, a fixed-effects model was used for the meta-analysis; otherwise, a random-effects model was used. The odds ratios (ORs) and 95\% confidence intervals (CIs) were calculated, and pooled ORs for each SNP were obtained from the meta-analysis using comparability analysis. The Hardy-Weinberg equilibrium (HWE) in controls was achieved by using a $\chi^{2}$ test, and $p$ value less than 0.05 suggested obvious deviation. A leave-one-out sensitivity analysis was conducted to explore the effect of each study on the pooled ORs. A funnel plot of publication bias was evaluated by using Begg's funnel plots [23] and Egger's linear regression test [24]. Subgroup analysis was performed based on ethnicity and the disease subtype. Meta-regression by the restricted maximum likelihood approach was utilized to determine the potential source of heterogeneity in all populations. A $p$ value of $<0.05$ was considered statistically significant in a two-tailed $T$ test. The software Stata 12.0 (StataCorp LP, TX, USA) and R programming language were used for comprehensive meta-analyses. All results were checked separately by 2 reviewers (J.Z. and P.Z.).

\section{Results}

\section{Literature Search}

A detailed selection process for eligible studies was illustrated in Figure 1. A total of 143 articles were identified in the above electronic databases. Of these, 116 articles were excluded because 102 were duplicates, and 14 did not investigate the relationship between PTPN22 and CTLA-4 polymorphisms and the risk of uveitis. The remaining 27 records were eligible for inclusion in the study. Among these studies, one had depicted other PTPN22 polymorphisms with VKH disease, and another six did not contain enough genotypic data. Finally, 20 published records with 5,320 cases and 8,839 controls were included in our meta-analysis $[13,16,25-42]$. 


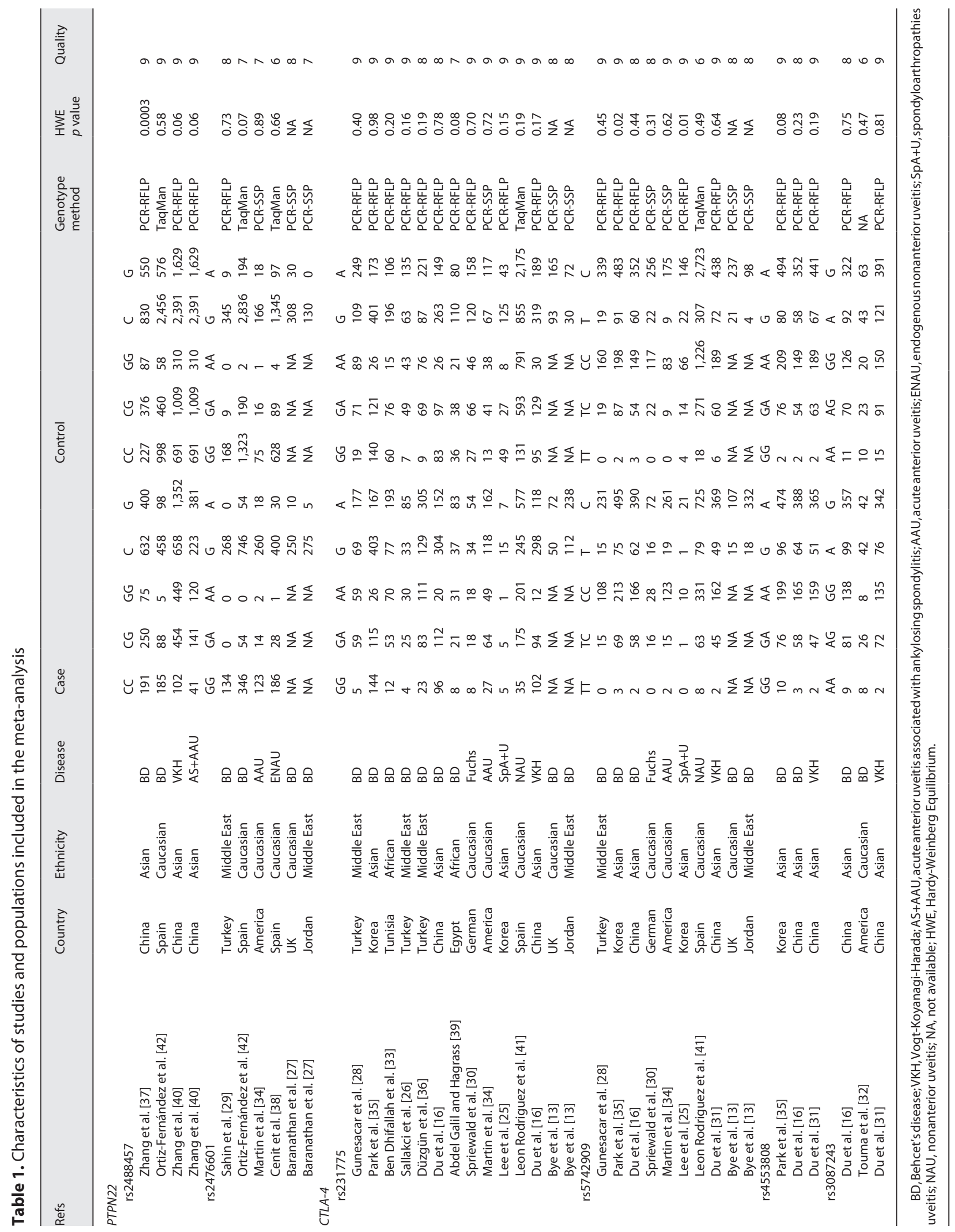


Table 2. Heterogeneity statistics and publication bias analysis

\begin{tabular}{|c|c|c|c|c|c|c|c|}
\hline \multirow[t]{2}{*}{ SNP } & \multirow[t]{2}{*}{ Polymorphism } & \multirow[t]{2}{*}{ No. } & \multicolumn{3}{|c|}{ Test of heterogeneity } & \multicolumn{2}{|c|}{ Publication bias } \\
\hline & & & $R^{2}, \%$ & $p$ value & model & Begg's test & Egger's test \\
\hline \multicolumn{8}{|l|}{ PTPN22 } \\
\hline \multirow[t]{5}{*}{ rs2488457 } & C versus G (allele) & 4 & 70.1 & 0.018 & $\mathrm{R}$ & 0.734 & 0.176 \\
\hline & CC versus GG (homozygote) & 4 & 72.5 & 0.012 & $\mathrm{R}$ & 1.000 & 0.808 \\
\hline & CG versus GG (heterozygote) & 4 & 66.6 & 0.029 & $\mathrm{R}$ & 1.000 & 0.871 \\
\hline & $\mathrm{CC}+\mathrm{CG}$ versus $\mathrm{GG}$ (dominant) & 4 & 73.0 & 0.011 & $\mathrm{R}$ & 1.000 & 0.975 \\
\hline & CC versus CG+GG (recessive) & 4 & 61.4 & 0.051 & $\mathrm{R}$ & 0.308 & 0.033 \\
\hline \multirow[t]{5}{*}{ rs2476601 } & G versus A (allele) & 6 & 56.5 & 0.043 & $\mathrm{R}$ & 0.452 & 0.359 \\
\hline & GG versus AA (homozygote) & 4 & 0.0 & 0.965 & $\mathrm{~F}$ & 1.000 & 0.810 \\
\hline & GA versus AA (heterozygote) & 4 & 0.0 & 0.781 & $\mathrm{~F}$ & 1.000 & 0.928 \\
\hline & $G G+G A$ versus $A A$ (dominant) & 4 & 0.0 & 0.947 & $\mathrm{~F}$ & 1.000 & 0.829 \\
\hline & GG versus $\mathrm{GA}+\mathrm{AA}$ (recessive) & 4 & 49.6 & 0.114 & $\mathrm{~F}$ & 0.089 & 0.036 \\
\hline \multicolumn{8}{|c|}{ - } \\
\hline \multirow[t]{5}{*}{ rs231775 } & G versus $A$ (allele) & 14 & 87.8 & $<0.001$ & $\mathrm{R}$ & 0.037 & 0.289 \\
\hline & GG versus AA (homozygote) & 12 & 87.0 & $<0.001$ & $\mathrm{R}$ & 0.244 & 0.399 \\
\hline & GA versus AA (heterozygote) & 12 & 77.0 & $<0.001$ & $\mathrm{R}$ & 0.193 & 0.336 \\
\hline & GG+GA versus $A A$ (dominant) & 12 & 85.1 & $<0.001$ & $\mathrm{R}$ & 0.244 & 0.344 \\
\hline & GG versus $G A+A A$ (recessive) & 12 & 80.2 & $<0.001$ & $\mathrm{R}$ & 0.193 & 0.173 \\
\hline \multirow[t]{5}{*}{ rs5742909 } & T versus C (allele) & 10 & 36.4 & 0.117 & $\mathrm{~F}$ & 0.592 & 0.258 \\
\hline & TT versus CC (homozygote) & 8 & 0.0 & 0.611 & $\mathrm{~F}$ & 1.000 & 0.540 \\
\hline & TC versus CC (heterozygote) & 8 & 41.9 & 0.121 & $\mathrm{~F}$ & 0.389 & 0.316 \\
\hline & $T T+T C$ versus $C C$ (dominant) & 8 & 43.7 & 0.087 & $\mathrm{~F}$ & 0.386 & 0.392 \\
\hline & TT versus TC+CC (recessive) & 8 & 0.0 & 0.613 & $\mathrm{~F}$ & 1.000 & 0.556 \\
\hline \multirow[t]{5}{*}{ rs4553808 } & G versus $A$ (allele) & 3 & 0.0 & 0.451 & $\mathrm{~F}$ & 0.296 & 0.107 \\
\hline & GG versus AA (homozygote) & 3 & 0.0 & 0.384 & $\mathrm{~F}$ & 0.296 & 0.179 \\
\hline & GA versus AA (heterozygote) & 3 & 0.0 & 0.844 & $\mathrm{~F}$ & 0.296 & 0.322 \\
\hline & $\mathrm{GG}+\mathrm{GA}$ versus $\mathrm{AA}$ (dominant) & 3 & 0.0 & 0.653 & $\mathrm{~F}$ & 0.296 & 0.201 \\
\hline & GG versus $G A+A A$ (recessive) & 3 & 0.0 & 0.399 & $\mathrm{~F}$ & 0.296 & 0.186 \\
\hline \multirow[t]{5}{*}{ rs3087243 } & A versus $G$ (allele) & 3 & 58.7 & 0.089 & $\mathrm{R}$ & 1.000 & 0.403 \\
\hline & AA versus GG (homozygote) & 3 & 72.0 & 0.028 & $\mathrm{R}$ & 1.000 & 0.729 \\
\hline & AG versus GG (heterozygote) & 3 & 56.7 & 0.099 & $\mathrm{R}$ & 0.296 & 0.167 \\
\hline & $A A+A G$ versus $G G$ (dominant) & 3 & 63.4 & 0.065 & $\mathrm{R}$ & 0.296 & 0.258 \\
\hline & AA versus $A G+G G$ (recessive) & 3 & 55.5 & 0.106 & $\mathrm{~F}$ & 1.000 & 0.326 \\
\hline
\end{tabular}

No., number of studies; F, fixed-effects model; R, random-effects model.

\section{General Characteristics of the Included Studies}

Various types of uveitis including BD, VKH, acute anterior uveitis, nonanterior uveitis, acute anterior uveitis associated with ankylosing spondylitis, endogenous nonanterior uveitis, and spondyloarthropathies uveitis were included in our research. The characteristics of each study were presented in Table 1. Different genotyping methods, including polymerase chain reaction-restriction fragment length polymorphism, polymerase chain reactionsequence-specific primer, and TaqMan SNP genotyping, assays were applied. The genotypic distribution from all studies was in agreement with the HWE $(p>0.05)$ except for 3 studies [25, 35, 37]. The quality assessments for all selected articles are manifested in Table 1. Furthermore, all studies were of high quality (i.e., they were awarded more than 6 stars) according to the Newcastle-Ottawa scale.

\section{Association between PTPN22 Gene Polymorphisms and Uveitis}

Totally, ten eligible studies that described common SNPs of the PTPN22 gene (rs2488457 and rs2476601) association with uveitis were included in our meta-analysis. Analysis of the association of rs 2488457 polymorphism and uveitis susceptibility was based on 4 studies. As delineated in Table 2 , significant heterogeneity was identified in all genotypic models $(p<0.1)$, and a random-effects model was applied. A significantly positive relation- 


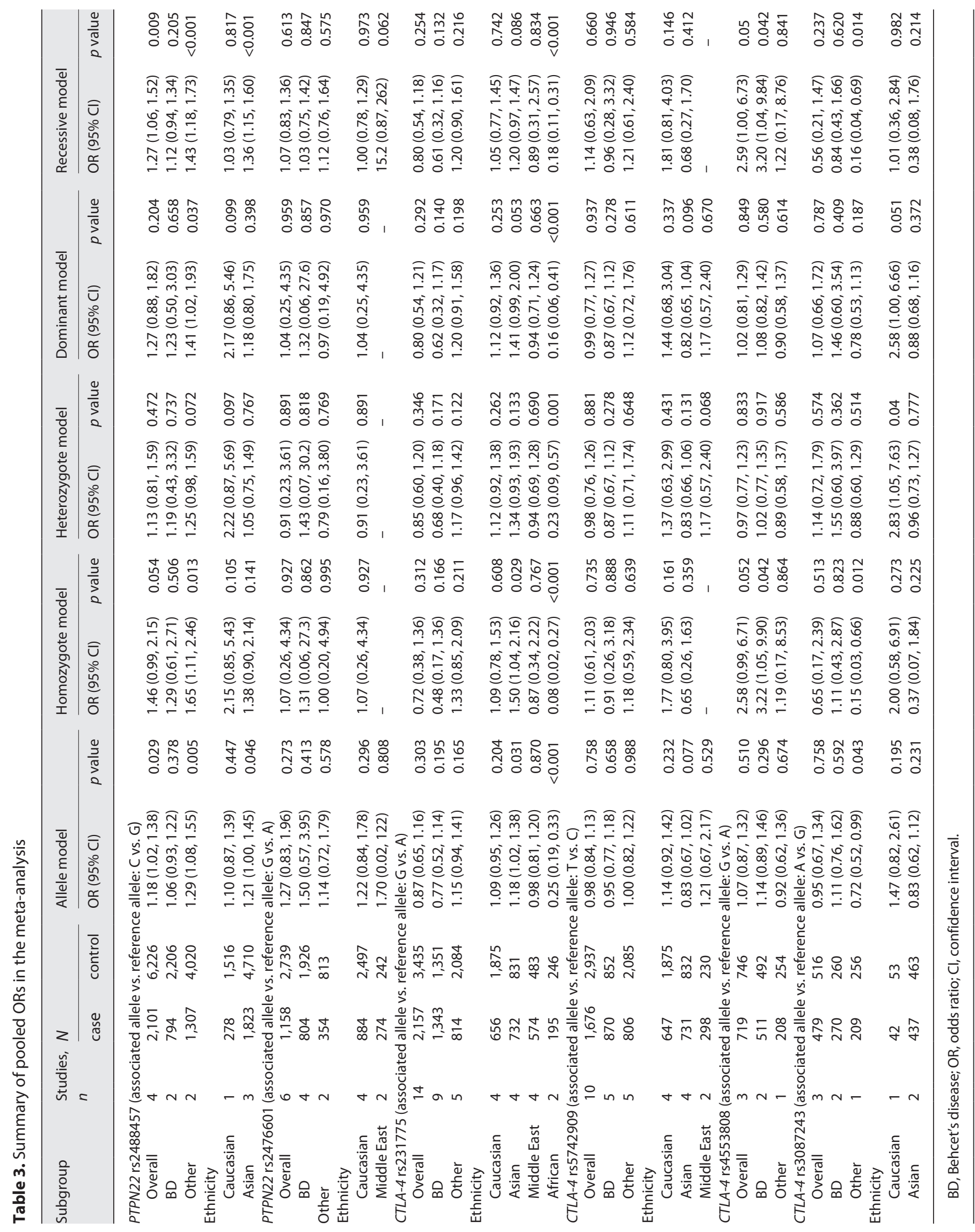




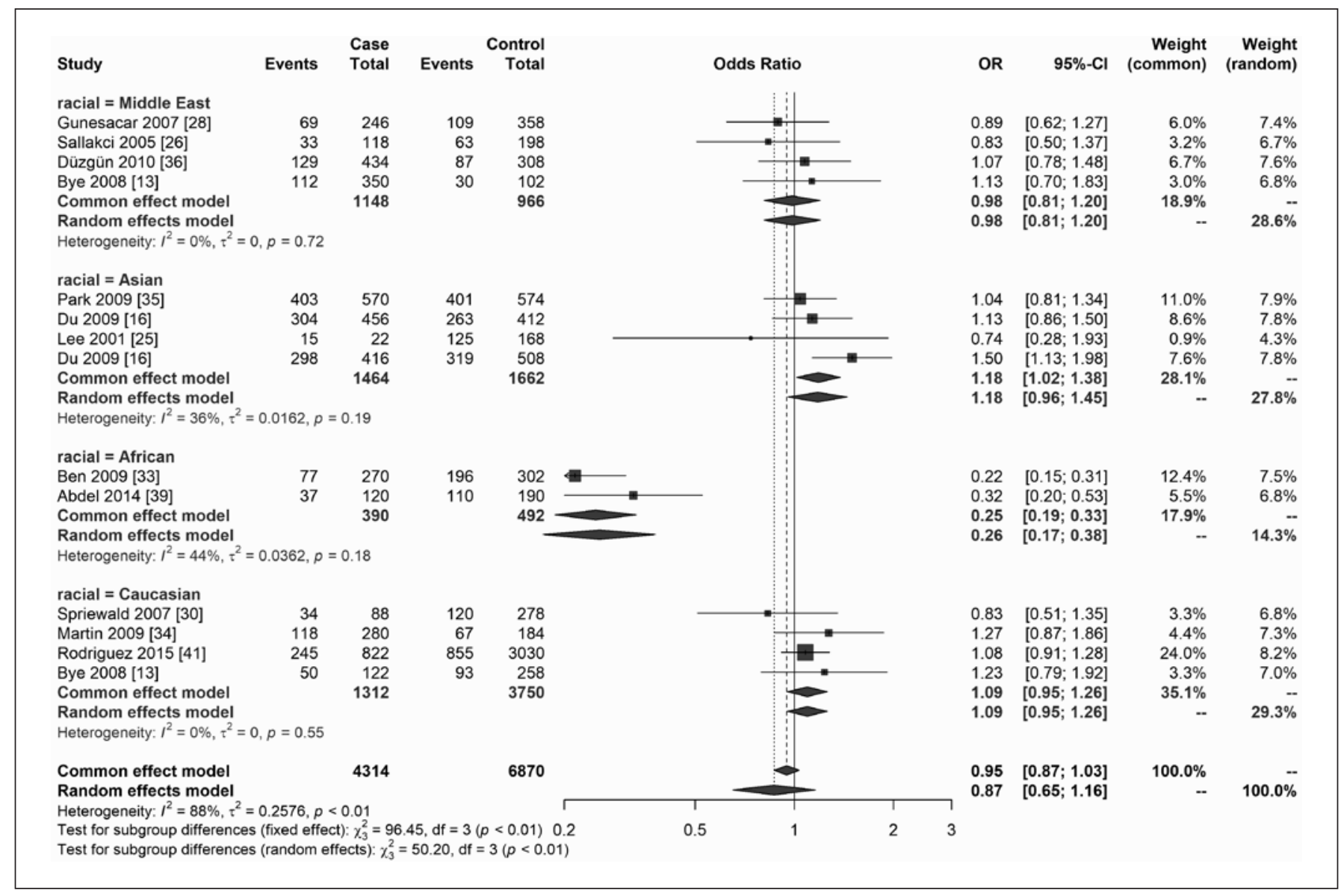

Fig. 2. Forest plots of studies reporting the associations between CTLA-4 rs 231775 and risk of uveitis. Each square indicates a study, and the area of squares is proportional to the weight of the study. The diamond represents the summary OR and 95\% CI.

ship was observed between rs2488457 polymorphisms and uveitis susceptibility ( $\mathrm{C}$ vs. $\mathrm{G}, \mathrm{OR}=1.18,95 \% \mathrm{CI}=$ $1.02-1.38, p=0.029$ and $\mathrm{CC}$ vs. $\mathrm{CG}+\mathrm{GG}, \mathrm{OR}=1.27,95 \%$ $\mathrm{CI}=1.06-1.52, p=0.009$; Table 3 ). In addition, disease type-stratified analysis demonstrated that the $\mathrm{C}$ allele was not correlated with greater prevalence of BD in any model $(p>0.05)$ but with other uveitis subtypes (C vs. G, OR $=1.29,95 \% \mathrm{CI}=1.08-1.55, p=0.005 ; \mathrm{CC}$ vs. GG, $\mathrm{OR}=$ $1.65,95 \% \mathrm{CI}=1.11-2.46, p=0.013 ; \mathrm{CC}+\mathrm{CG}$ vs. GG, $\mathrm{OR}$ $=1.41,95 \% \mathrm{CI}=1.02-1.93, p=0.037$; and $\mathrm{CC}$ vs. $\mathrm{CG}+\mathrm{GG}$, $\mathrm{OR}=1.43,95 \% \mathrm{CI}=1.18-1.73, p<0.001$ ). Subsequently, in terms of the subgroup analysis of the ethnicity, $C$ allele conferred significantly increased susceptibility to uveitis in the Asian region ( $\mathrm{C}$ vs. $\mathrm{G}, \mathrm{OR}=1.21,95 \% \mathrm{CI}=1.00$ $1.45, p=0.046$ and $\mathrm{CC}$ vs. $\mathrm{CG}+\mathrm{GG}, \mathrm{OR}=1.36,95 \% \mathrm{CI}=$ $1.15-1.60, p<0.001)$.

Moreover, the pooled results between PTPN22 rs2476601 and uveitis risk declined the presence of sig- nificant association in our meta-analysis $(p>0.05$, Table 3).

\section{Association between CTLA-4 Gene Polymorphisms and Uveitis}

Four polymorphic sites (rs231775, rs5742909, rs4553808, and rs3087243) in the CTLA-4 gene were commonly reported to investigate potential susceptibility to uveitis. For CTLA-4 rs231775, fourteen studies in thirteen articles were enrolled and reanalyzed. Obvious heterogeneity was detected in the genetic models; thus, a random-effects model was used (Table 2). There was no evidence of association between rs231775 and uveitis in general for any model ( $p>0.05$, Table 3$)$. Subgroup analysis based on ethnicity also did not reveal any risk of uveitis among Caucasian and Middle-East regions. A marginally increased risk of uveitis was discovered in the Asian group (G vs. A, OR $=1.18,95 \% \mathrm{CI}=1.02-1.38, p=$ 
Fig. 3. Leave-one-out sensitivity analysis of the association between CTLA-4 rs231775 and risk of uveitis. The three vertical lines indicate the summary effect and its $95 \%$ CI. Each horizontal line shows the summary effect and its 95\% CI after elimination of the included study in turn.

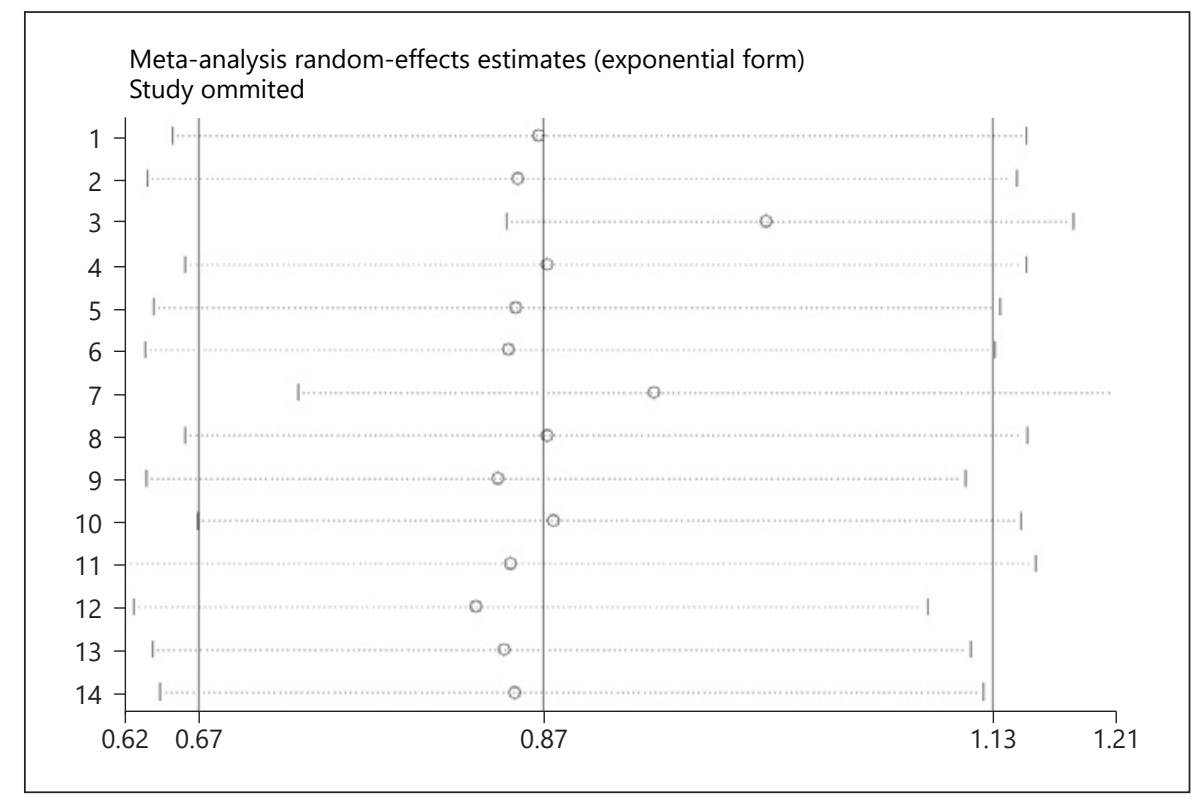

0.031 and GG vs. $\mathrm{AA}, \mathrm{OR}=1.50,95 \% \mathrm{CI}=1.04-2.16, p=$ $0.029)$. However, a significantly negative correlation was observed in the Africa group ( $\mathrm{G}$ vs. A, OR $=0.25,95 \% \mathrm{CI}$ $=0.19-0.33, p<0.001$, Fig. 2 ; GG vs. AA, OR $=0.08,95 \%$ $\mathrm{CI}=0.02-0.27, p<0.001$; GA vs. $\mathrm{AA}, \mathrm{OR}=0.23,95 \% \mathrm{CI}$ $=0.09-0.57, p<0.001 ; \mathrm{GG}+\mathrm{GA}$ vs. $\mathrm{AA}, \mathrm{OR}=0.16,95 \%$ $\mathrm{CI}=0.06-0.41, p<0.001$; and $\mathrm{GG}$ vs. $\mathrm{GA}+\mathrm{AA}, \mathrm{OR}=0.18$, $95 \% \mathrm{CI}=0.11-0.31, p<0.001$; Table 3 ).

Meta-analysis of ten studies was performed to investigate the association of rs5742909 polymorphism with uveitis. A pooled result of all genetic models implied that there was no obvious correlation among them $(p>0.05$, Table 3). Subgroup analysis based on ethnicity and the disease type also failed to find any relationship (Table 3 ).

Only three studies were focused on CTLA-4 rs4553808 with uveitis in Asian populations. No evidence of between-study heterogeneity under the five genetic models was observed, and a fixed-effects model was added $(p>$ 0.1 , Table 2). Subgroup analysis based on the disease type revealed that the rs 4553808 GG genotype conferred an elevated risk of $\mathrm{BD}(\mathrm{GG}$ vs. $\mathrm{AA}, \mathrm{OR}=3.22,95 \% \mathrm{CI}=$ $1.05-9.90, p=0.042$ and $\mathrm{GG}$ vs. $\mathrm{GA}+\mathrm{AA}, \mathrm{OR}=3.20,95 \%$ $\mathrm{CI}=1.04-9.84, p=0.042$ ).

For CTLA-4 rs3087243 G>A polymorphism, 3 studies examined the predisposition to uveitis in Caucasian and Asian populations. Overall, the A allele could not convey any risk of uveitis in all genetic models $(p>0.05)$. Subgroup analysis based on ethnicity suggested a positive relationship between the heterozygote model and Cauca- sian region (AG vs. $\mathrm{GG}, \mathrm{OR}=2.83,95 \% \mathrm{CI}=1.05-7.63$, $p=0.04)$.

\section{Sensitivity Analysis}

In the sensitivity analysis of the effect of each study on the pooled ORs, each study was omitted in turn. Although 3 studies $[25,35,37]$ showed evident deviation from the HWE $(p<0.05)$, excluding each of the studies in turn did not affect the pooled ORs (rs231775 G vs. A, Fig. 3), which inferred that the combined results of present meta-analysis were relatively robust.

\section{Publication Bias}

A series of Begg's funnel plots and Egger's regression tests were applied to examine potential publication bias in the studies of the association of the PTPN22 and CTLA4 polymorphisms with uveitis. As shown in Table 2, no obvious publication biases were discovered, with the shape of the funnel plots of each polymorphism site nearly symmetrical (rs231775 G vs. A, Fig. 4).

\section{Meta-Regression Analysis}

Significant heterogeneity was identified for our model comparisons, so meta-regression analysis was used to explore the potential heterogeneity in turn. The ethnicity, disease type, genotypic method, and sample size were thoroughly regarded as independent covariate factors. As delineated in Table 4, meta-regression indicated that the disease type was the source of between-study heterogene- 
Fig. 4. Begg's funnel plot of the association between CTLA-4 rs231775 and risk of uveitis. The $x$-axis is the SE of $\log (\mathrm{OR})$, and the $y$-axis is the natural logarithm of OR. SE, standard error.

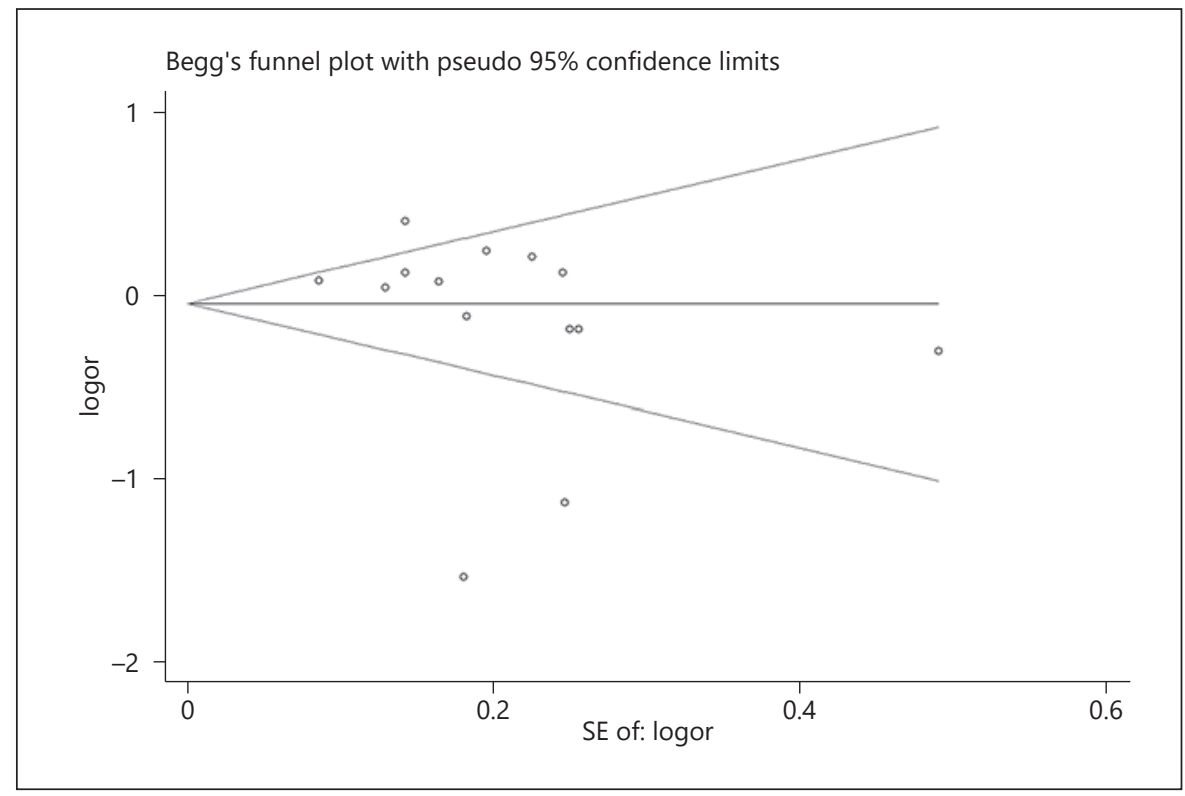

ity (95\% CI $=1.73-2.14, p=0.003)$ in rs231775. Simultaneously, we found that none of the other potential confounding factors contributed to the source of heterogeneity.

\section{Discussion}

In this systematic meta-analysis of 20 case-control studies, 6 SNPs from PTPN22 and CTLA-4 gene were delineated to investigate the association with uveitis. Three variants were shared across uveitis and could confer genetic predisposition to uveitis.

PTPN22 belongs to a family of genes encoding protein tyrosine phosphatases, which play a negative role in the TCR signal pathway via dephosphorylation of protein tyrosine kinases. These kinases, which contain the Src family (Lck and Fyn being the most important) and the Syk family (ZAP-70 being the most important), cause phosphorylation of immunoreceptor tyrosine-based activation motifs (ITAMs). ITAMs of the TCR-associated CD3区-chain induce several intracellular signaling pathways [43]. Functional studies derived from human and mouse cells indicated that PTPN22 knockdown or pharmacological inhibition could enhance TCR signaling [44-46].

Although increasing studies have investigated the correlation between PTPN22 genetic mutation and autoimmune diseases, the functional consequences of a single
Table 4. Meta-regression for the heterogeneity of PTPN22 and CTLA-4 genetic polymorphisms

\begin{tabular}{lllll}
\hline Covariate factor & Exp (b) & Std. err & $p$ value & $95 \% \mathrm{Cl}$ \\
\hline PTPN22 rs2488457 & & & & \\
$\quad$ Disease type & 0.82 & 0.09 & 0.210 & $0.51-1.32$ \\
$\quad$ Ethnicity & 0.91 & 0.18 & 0.681 & $0.38-2.16$ \\
$\quad$ Genotypic method & 0.91 & 0.18 & 0.681 & $0.38-2.16$ \\
$\quad$ Cases, $n$ & 1.00 & 0.0001 & 0.165 & $0.99-1.00$ \\
PTPN22 rs2476601 & & & & \\
$\quad$ Disease type & 0.84 & 0.54 & 0.796 & $0.14-5.05$ \\
$\quad$ Ethnicity & 0.71 & 1.03 & 0.826 & $0.01-39.76$ \\
Genotypic method & 0.08 & 0.13 & 0.203 & $0.0008-8.04$ \\
$\quad$ Cases, $n$ & 0.99 & 0.002 & 0.302 & $0.99-1.00$ \\
CTLA-4 rs231775 & & & & \\
$\quad$ Disease type & 1.26 & 0.73 & 0.003 & $1.73-2.14$ \\
$\quad$ Ethnicity & 0.70 & 0.23 & 0.300 & $0.35-1.43$ \\
$\quad$ Genotypic method & 1.53 & 0.48 & 0.202 & $0.77-3.04$ \\
Cases, $n$ & 1.00 & 0.001 & 0.242 & $0.99-1.00$ \\
CTLA-4 rs3087243 & & & & \\
$\quad$ Disease type & 0.65 & 0.19 & 0.388 & $0.01-30.5$ \\
$\quad$ Ethnicity & 1.75 & 0.63 & 0.361 & $0.02-165.2$ \\
$\quad$ Genotypic method & 1.75 & 0.63 & 0.361 & $0.02-165.2$ \\
$\quad$ Cases, $n$ & 0.99 & 0.002 & 0.415 & $0.97-1.03$ \\
\hline
\end{tabular}

Exp, exponential; Std. err, standard error; $\mathrm{Cl}$, confidence interval.

base change at the DNA level remain to be clarified. The present meta-analysis examined the literature on two SNPs (rs2488457 and rs2476601) of the PTPN22 gene with uveitis. PTPN22 rs2488457 is located in the promot- 
er region containing the core motif of the transcription factor AP-4 binding consensus sequence. Our meta-analysis was the first to confirm the association between rs2488457 and uveitis risk. Especially, the C allele was a risk factor for other autoimmune uveitis types and in the Asian group but not BD. The inconsistency may be attributed to different immune-mediated pathways in these uveitis entities. It has been commonly believed that $\mathrm{BD}$ is considered as an autoinflammatory disease, which differs from other type of uveitis $[47,48]$. The common PTPN22 polymorphism rs2476601 $(1858 \mathrm{C}>\mathrm{T})$ encodes an arginine-to-tryptophan substitution (amino acid 620, Arg620 Trp) in the first proline-rich motif of the PTPN22 protein. Trp620 is known to prevent LYP from binding to at least one of its adapter molecules such as Csk kinase. Thus, LYP containing Trp instead of Arg at this position cannot complex with Csk so that abrogates inhibition of T-cell activation [49]. The failure of T-cell inhibition following an immune attack on the body could lead to extensive Tcell proliferation and autoimmune phenotype ultimately, which would be potentially fatal [50]. This hypothesis was confirmed by a comprehensive meta-analysis of 12 autoimmune diseases, in which rs2476601C $>$ T was strongly correlated with rheumatoid arthritis and systemic lupus erythematosus risk [19]. However, the pooled ORs of PTPN22 rs2476601 did not imply any association with uveitis in our current meta-analysis. The remarkable difference between our data and the earlier meta-analyses may be explained by the disease-specific nature.

The gene CTLA-4, located on chromosome $2 \mathrm{q} 33$, is a crucial negative regulator of T-cell activation. However, the exact mechanisms involved in how CTLA-4 restrains T-cell activation are still not confirmed. One hypothesis suggested that CTLA-4 might successfully compete with CD28 for its CD80/86 ligands. CD28, which is constitutively expressed on the surface of $\mathrm{T}$ cells, plays an important role in signaling following $\mathrm{T}$-cell binding to antigenpresenting cells [51]. Thus, combining with CD80/86 ligands, CTLA-4 could inhibit the costimulatory effect of CD28 and T-cell activation. A second hypothesis proposed that CTLA-4 might interact with Lck, Fyn, and ZAP-70 protein kinases through SHP1 phosphatases and exert its inhibition effect by acting on downstream signal pathways [52]. According to a third hypothesis, CTLA-4 could directly interact with TCR ITAM and disrupt the cascade of biochemical signals that lead to activation of $\mathrm{T}$ cells [53]. Lastly, a recent study showed that CTLA-4 could regulate the $\mathrm{Cb} 1$ family molecule as a possible mechanism for aberrant T-cell activation for the occurrence of autoimmunity [54].

PTPN22 and CTLA-4 Genetic Variants with Uveitis
The present meta-analysis investigated the potential correlation between CTLA-4 genetic variants and uveitis. Combined results of rs231775 polymorphism revealed a reduced trend toward uveitis in an ethnic stratification analysis. Additionally, our combined results revealed significantly positive association between rs4553808 and uveitis risk. These results differ from previous meta-analysis, which did not find any positive relationship $[17,18]$. This discrepancy could be attributed to much more studies and larger populations. Therefore, it is certain that these findings are more accurate than before.

In our meta-analysis, further subgroup analysis based on ethnicity indicated distinctive associations between rs 2488457 and rs 231775 polymorphisms and uveitis. The reasons for this discrepancy could be explained by genetic backgrounds with different allele frequency among populations, living environments, and matching criteria. Besides, the results of our meta-regression analysis have shown that the disease type may be the major source of heterogeneity between studies, but ethnicity, genotypic method, and sample size could not explain obvious heterogeneity, suggesting that these confounding factors may not cause statistical bias. These disparate conclusions may be attributed by the relatively small sample size, resulting in substantial estimation errors.

There are several limitations in this meta-analysis. First, the subgroup analysis revealed disease type-induced heterogeneity in the association of CTLA-4 rs231775 with uveitis. However, the overall effect cannot be affected in the examination of sensitivity analysis and meta-regression analysis. Second, the sample size in each study was relatively small. Further records with much larger populations and different ethnic groups are encouraged. Third, the ethnic origins from patients and controls were not specified in all the studies, and different criteria were used for the inclusion of controls, which may result in potential heterogeneity. Fourth, the current research should be registered in the PROSPERO or Cochrane system. We have tried our best and failed to register as soon as possible, which could result in bias. But our meta-analysis was performed strictly in accordance with the process of systematic review. Fifth, the information of sex differences was not available in uveitis subtypes, thus the potential heterogeneity by sex could not be evaluated. Finally, all eligible studies were retrieved from English and Chinese language records. This would result in selection bias in this meta-analysis. 


\section{Conclusion}

In summary, the results of this comprehensive metaanalysis demonstrated that PTPN22 rs2488457 conferred strong susceptibility to uveitis, especially in the Asian region. The CTLA-4 rs231775 polymorphism convey potential protection against uveitis in African populations; otherwise, CTLA-4 rs4553808 displayed an elevated risk of $\mathrm{BD}$. Although our results revealed PTPN22 and CTLA4 could be prioritizing candidate genes for uveitis, further well-designed independent studies with larger case-control individuals are required to validate these findings.

\section{Acknowledgements}

This manuscript does not include any nonauthor contributors to acknowledge.

\section{Statement of Ethics}

All analyses were based on previous published studies; thus, no ethical approval and patient consent are required.

\section{Funding Sources}

This work was supported by the National Natural Science Foundation Project (grant number 81770916, 81400389) and Chongqing Science \& Technology Foundation Project (grant number cstc2014jcyjA10111). No funding bodies had any role in study design, data collection and analysis, decision to publish, or preparation of the manuscript.

\section{Author Contributions}

J.Q. conceived the study. J.Z. and P.Z. performed and checked the available information from eligible articles in this meta-analysis. P.Z. analyzed the data. S.H. prepared Figures 1-4 and Tables 1-4. J.Z. wrote the main manuscript text. J.Q. reviewed and revised the manuscript. All the authors read and approved the final manuscript.

\section{Data Availability Statement}

All data generated or analyzed during this study are included in this article and its online supplementary material. Further inquiries can be directed to the corresponding author.

\section{Conflict of Interest Statement}

The authors declare that they have no conflicts of interest to this work.

\section{References}

1 Yang $\mathrm{P}$, Zhang Z, Zhou H, Li B, Huang X, Gao $\mathrm{Y}$, et al. Clinical patterns and characteristics of uveitis in a tertiary center for uveitis in China. Curr Eye Res. 2005 Nov;30(11):943-8.

2 Hikita S, Sonoda KH, Hijioka K, Fujimoto T, Ito $\mathrm{T}$, Ishibashi $\mathrm{T}$. [Incidence of uveitis in the northern Kyushu region of Japan: comparison between the periods of 1996-2001 and 2003-2008]. Nippon Ganka Gakkai zasshi. 2012 Sep;116(9):847-55.

3 Walton RC, Ashmore ED. Retinal vasculitis. Curr Opin Ophthalmol. 2003 Dec;14(6):4139.

4 Yamaki K, Gocho K, Hayakawa K, Kondo I, Sakuragi S. Tyrosinase family proteins are antigens specific to Vogt-Koyanagi-Harada disease. J Immunol. 2000 Dec 15;165(12):73239.

5 Piga M, Mathieu A. Genetic susceptibility to Behcet's disease: role of genes belonging to the MHC region. Rheumatology. 2011 Feb; 50(2):299-310.
6 Brewerton DA, Hart FD, Nicholls A, Caffrey $\mathrm{M}$, James DC, Sturrock RD. Ankylosing spondylitis and HL-A 27. Lancet. 1973 Apr 28; 1(7809):904-7.

7 Hou S, Kijlstra A, Yang P. Molecular genetic advances in uveitis. Prog Mol Biol Transl Sci. 2015;134:283-98.

8 Moorthy RS, Inomata H, Rao NA. Vogt-Koyanagi-Harada syndrome. Surv Ophthalmol. 1995 Jan-Feb;39(4):265-92.

9 Koarada S, Haruta Y, Tada Y, Ushiyama O, Morito F, Ohta A, et al. Increased entry of CD4+ T cells into the Th1 cytokine effector pathway during $\mathrm{T}$-cell division following stimulation in Behcet's disease. Rheumatology. 2004 Jul;43(7):843-51.

10 Tarner IH, Fathman CG. Does our current understanding of the molecular basis of immune tolerance predict new therapies for autoimmune disease? Nat Clin Pract Rheumatol. 2006 Sep;2(9):491-9.

11 Cohen S, Dadi H, Shaoul E, Sharfe N, Roifman CM. Cloning and characterization of a lymphoid-specific, inducible human protein tyrosine phosphatase, Lyp. Blood. 1999 Mar 15;93(6):2013-24.
12 Rawlings DJ, Dai X, Buckner JH. The role of PTPN22 risk variant in the development of autoimmunity: finding common ground between mouse and human. J Immunol. 2015 Apr 1;194(7):2977-84.

13 Bye L, Modi N, Stanford MR, Kondeatis E, Vaughan R, Fortune F, et al. CTLA-4 polymorphisms are not associated with ocular inflammatory disease. Tissue Antigens. 2008 Jul;72(1):49-53.

14 Sharpe AH, Freeman GJ. The B7-CD28 superfamily. Nat Rev Immunol. 2002 Feb;2(2): 116-26.

15 Berg M, Zavazava N. Regulation of CD28 expression on CD8+ T cells by CTLA-4. J Leukoc Biol. 2008 Apr;83(4):853-63.

16 Du L, Yang P, Hou S, Zhou H, Kijlstra A. No association of CTLA-4 polymorphisms with susceptibility to Behçet disease. Br J Ophthalmol. 2009 Oct;93(10):1378-81.

17 Zhang YJ, Xu WD, Duan ZH, Liu SS, Pan HF, Ye DQ. Lack of association between CTLA-4 $+49 \mathrm{~A} / \mathrm{G}$ and $-318 \mathrm{C} / \mathrm{T}$ polymorphisms and Behcet's disease risk: a meta-analysis. Clin Exp Rheumatol. 2012 May-Jun;30(3 Suppl 72):S46-50. 
18 Lee YH, Song GG. CTLA-4 polymorphisms and susceptibility to Behcet's disease: a metaanalysis. Mol Biol Rep. 2012 Sep;39(9):90415.

19 Zheng J, Ibrahim S, Petersen F, Yu X. Metaanalysis reveals an association of PTPN22 C1858T with autoimmune diseases, which depends on the localization of the affected tissue. Genes Immun. 2012 Dec;13(8):641-52.

20 Moher D, Liberati A, Tetzlaff J, Altman DG; PRISMA Group. Preferred reporting items for systematic reviews and meta-analyses: the PRISMA statement. BMJ. 2009 Jul 21;339(7): b2535.

21 Zhai Y, Xu K, Huang F, Peng H, Feng CC, Zhu $\mathrm{KK}$, et al. Association of interleukin 23 receptor gene polymorphisms (rs10489629, rs7517847) with rheumatoid arthritis in European population: a meta-analysis. Mol Biol Rep. 2012 Sep;39(9):8987-94.

22 Higgins JP, Thompson SG. Quantifying heterogeneity in a meta-analysis. Stat Med. 2002 Jun 15;21(11):1539-58.

23 Begg CB, Mazumdar M. Operating characteristics of a rank correlation test for publication bias. Biometrics. 1994 Dec;50(4):1088-101.

24 Egger M, Davey Smith G, Schneider M, Minder C. Bias in meta-analysis detected by a simple, graphical test. BMJ. 1997 Sep 13; 315(7109):629-34.

25 Lee YH, Ji JD, Sohn J, Song GG. Polymorphsims of CTLA-4 exon $1+49$, CTLA-4 promoter -318 and Fas promoter -670 in spondyloarthropathies. Clin Rheumatol. 2001;20(6): $420-2$.

26 Sallakci N, Bacanli A, Coskun M, Yavuzer U, Alpsoy E, Yegin O. CTLA-4 gene 49A/G polymorphism in Turkish patients with Behçet's disease. Clin Exp Dermatol. 2005 Sep;30(5): 546-50.

27 Baranathan V, Stanford MR, Vaughan RW, Kondeatis E, Graham E, Fortune F, et al. The association of the PTPN22 620W polymorphism with Behcet's disease. Ann Rheum Dis. 2007 Nov;66(11):1531-3

28 Gunesacar R, Erken E, Bozkurt B, Ozer HT, Dinkci S, Erken EG, et al. Analysis of CD28 and CTLA-4 gene polymorphisms in Turkish patients with Behcet's disease. Int I Immunogenet. $2007 \mathrm{Feb}$;34(1):45-9.

29 Sahin N, Bicakcigil M, Atagunduz P, Direskeneli H, Saruhan-Direskeneli G. PTPN22 gene polymorphism in Behçet's disease. Tissue Antigens. 2007 Nov;70(5):432-4.

30 Spriewald BM, Lefter C, Huber I, Lauer B, Wenkel $\mathrm{H}$. A suggestive association of fuchs heterochromic cyclitis with cytotoxic $\mathrm{T}$ cell antigen 4 gene polymorphism. Ophthalmic Res. 2007;39(2):116-20.
31 Du L, Yang P, Hou S, Lin X, Zhou H, Huang $\mathrm{X}$, et al. Association of the CTLA-4 gene with Vogt-Koyanagi-Harada syndrome. Clin Immunol. 2008 Apr;127(1):43-8.

32 Touma Z, Hamdan A, Shamseddeen W, Nasr J, Makhoul N, Zalloua PA, et al. CTLA-4 gene variants are not associated with Behcet's disease or its clinical manifestations. Clin Exp Rheumatol. 2008 Jul-Aug;26(4 Suppl 50): S132.

33 Ben Dhifallah I, Chelbi H, Braham A, Hamzaoui K, Houman MH. CTLA-4 +49A/G polymorphism is associated with Behçet's disease in a Tunisian population. Tissue Antigens. 2009 Mar;73(3):213-7.

34 Martin TM, Bye L, Modi N, Stanford MR, Vaughan R, Smith JR, et al. Genotype analysis of polymorphisms in autoimmune susceptibility genes, CTLA-4 and PTPN22, in an acute anterior uveitis cohort. Mol Vis. 2009;15:20812.

35 Park KS, Baek JA, Do JE, Bang D, Lee ES. CTLA4 gene polymorphisms and soluble CTLA4 protein in Behcet's disease. Tissue Antigens. 2009 Sep;74(3):222-7.

36 Düzgün N, Duman T, Keskin O, Morris Y, Tutkak H. Cytotoxic T lymphocyte antigen-4 (CTLA-4) polymorphism in patients with Behcet's disease. Clin Exp Rheumatol. 2010 JulAug;28(4 Suppl 60):S100.

37 Zhang Q, Hou S, Jiang Z, Du L, Li F, Xiao X, et al. No association of PTPN22 polymorphisms with susceptibility to ocular Behcet's disease in two Chinese Han populations. PloS One. 2012;7(3):e31230.

38 Cenit MC, Marquez A, Cordero-Coma M, Fonollosa A, Llorenc V, Artaraz J, et al. Lack of association between the protein tyrosine phosphatase non-receptor type $22 \mathrm{R} 263 \mathrm{Q}$ and R620W functional genetic variants and endogenous non-anterior uveitis. Mol Vis. 2013;19:638-43.

39 Abdel Galil SM, Hagrass HA. The role of CTLA-4 exon-1 49 A/G polymorphism and soluble CTLA-4 protein level in egyptian patients with Behçet's disease. Biomed Res Int. 2014;2014:513915.

40 Zhang Q, Qi J, Hou S, Du L, Yu H, Cao Q, et al. A functional variant of PTPN22 confers risk for Vogt-Koyanagi-Harada syndrome but not for ankylosing spondylitis. PLoS One. 2014;9(5):e96943.

41 Leon Rodriguez DA, Serrano Lopera A, Cordero-Coma M, Marquez A, Fonollosa A, Ruiz-Arruza I, et al. Study of association of CTLA4 gene variants to non-anterior uveitis. Tissue Antigens. 2015 Nov;86(5):373-6.

42 Ortiz-Fernandez L, Montes-Cano MA, Garcia-Lozano JR, Conde-Jaldon M, Ortego-
Centeno N, Gonzalez-Leon R, et al. PTPN22 is not associated with Behcet's disease. Study spanning the complete gene region in the Spanish population and meta-analysis of the functional variant R620W. Clin Exp Rheumatol. 2016 Sep-Oct;34(6 Suppl 102):S41-5.

43 Stanford SM, Rapini N, Bottini N. Regulation of TCR signalling by tyrosine phosphatases: from immune homeostasis to autoimmunity. Immunology. 2012 Sep;137(1):1-19.

44 Stanford SM, Krishnamurthy D, Falk MD, Messina R, Debnath B, Li S, et al. Discovery of a novel series of inhibitors of lymphoid tyrosine phosphatase with activity in human $\mathrm{T}$ cells. J Med Chem. 2011 Mar 24;54(6):164054.

45 Brownlie RJ, Miosge LA, Vassilakos D, Svensson LM, Cope A, Zamoyska R. Lack of the phosphatase PTPN22 increases adhesion of murine regulatory $\mathrm{T}$ cells to improve their immunosuppressive function. Sci Signal. 2012 Nov 27;5(252):ra87.

46 Vang T, Liu WH, Delacroix L, Wu S, Vasile S, Dahl R, et al. LYP inhibits T-cell activation when dissociated from CSK. Nat Chem Biol. 2012 May;8(5):437-46.

47 Gul A. Behcet's disease as an autoinflammatory disorder. Curr Drug Targets Inflamm Allergy. 2005 Feb;4(1):81-3.

48 Greco A, Fusconi M, Gallo A, Turchetta R, Marinelli C, Macri GF, et al. Vogt-KoyanagiHarada syndrome. Autoimmun Rev. 2013 Sep;12(11):1033-8.

49 Gomez LM, Anaya JM, Martin J. Genetic influence of PTPN22 R620W polymorphism in tuberculosis. Hum Immunol. 2005 Dec; 66(12):1242-7.

50 Brand O, Gough S, Heward J. HLA, CTLA-4 and PTPN22: the shared genetic master-key to autoimmunity? Expert Rev Mol Med. 2005 Oct 17;7(23):1-15.

51 Egen JG, Allison JP. Cytotoxic T lymphocyte antigen- 4 accumulation in the immunological synapse is regulated by TCR signal strength. Immunity. 2002 Jan;16(1):23-35.

52 Marengere LE, Waterhouse P, Duncan GS, Mittrucker HW, Feng GS, Mak TW. Regulation of $\mathrm{T}$ cell receptor signaling by tyrosine phosphatase SYP association with CTLA-4. Science. 1996 May 24;272(5265):1170-3.

53 Lee KM, Chuang E, Griffin M, Khattri R, Hong DK, Zhang W, et al. Molecular basis of T cell inactivation by CTLA-4. Science. 1998 Dec 18;282(5397):2263-6.

54 Li D, Gal I, Vermes C, Alegre ML, Chong AS, Chen L, et al. Cutting edge: Cbl-b-one of the key molecules tuning CD28- and CTLA4-mediated T cell costimulation. J Immunol. 2004 Dec 15;173(12):7135-9. 